\title{
Far-from-Equilibrium Spin Transport in Heisenberg Quantum Magnets
}

\section{Citation}

Hild, Sebastian, Takeshi Fukuhara, Peter Schauß, Johannes Zeiher, Michael Knap, Eugene Demler, Immanuel Bloch, and Christian Gross. 2014. "Far-from-Equilibrium Spin Transport in Heisenberg Quantum Magnets." Physical Review Letters 113 (14) (October). doi:10.1103/ physrevlett.113.147205.

\section{Published Version}

10.1103/PhysRevLett.113.147205

\section{Permanent link}

http://nrs.harvard.edu/urn-3:HUL.InstRepos:22856734

\section{Terms of Use}

This article was downloaded from Harvard University's DASH repository, and is made available under the terms and conditions applicable to Other Posted Material, as set forth at http:// nrs.harvard.edu/urn-3:HUL.InstRepos:dash.current.terms-of-use\#LAA

\section{Share Your Story}

The Harvard community has made this article openly available.

Please share how this access benefits you. Submit a story.

\section{Accessibility}




\title{
Far-from-Equilibrium Spin Transport in Heisenberg Quantum Magnets
}

\author{
Sebastian Hild, ${ }^{1, *}$ Takeshi Fukuhara, ${ }^{1, \dagger}$ Peter Schauß, ${ }^{1}$ Johannes Zeiher, ${ }^{1}$ Michael Knap, ${ }^{2,3}$ Eugene Demler, ${ }^{2}$ \\ Immanuel Bloch, ${ }^{1,4}$ and Christian Gross ${ }^{1}$ \\ ${ }^{1}$ Max-Planck-Institut für Quantenoptik, 85748 Garching, Germany \\ ${ }^{2}$ Department of Physics, Harvard University, Cambridge, Massachusetts 02138, USA \\ ${ }^{3}$ ITAMP, Harvard-Smithsonian Center for Astrophysics, Cambridge, Massachusetts 02138, USA \\ ${ }^{4}$ Ludwig-Maximilians-Universität, Fakultät für Physik, 80799 München, Germany
}

(Received 25 July 2014; published 3 October 2014)

\begin{abstract}
We study experimentally the far-from-equilibrium dynamics in ferromagnetic Heisenberg quantum magnets realized with ultracold atoms in an optical lattice. After controlled imprinting of a spin spiral pattern with an adjustable wave vector, we measure the decay of the initial spin correlations through singlesite resolved detection. On the experimentally accessible time scale of several exchange times, we find a profound dependence of the decay rate on the wave vector. In one-dimensional systems, we observe diffusionlike spin transport with a dimensionless diffusion coefficient of $0.22(1)$. We show how this behavior emerges from the microscopic properties of the closed quantum system. In contrast to the onedimensional case, our transport measurements for two-dimensional Heisenberg systems indicate anomalous superdiffusion.
\end{abstract}

Since its introduction, the Heisenberg spin model has posed fundamental challenges for the understanding of nonequilibrium dynamics in quantum magnets. On a very basic, phenomenological level, the concept of spin diffusion was introduced more than 60 years ago [1-3]. It has been commonly applied to interpret nuclear magnetic resonance spin lattice relaxation and electron spin resonance experiments [4-8]. However, up to now it has never been justified $a b$ initio from a microscopic model. Moreover, many analytical and numerical studies suggested the existence of anomalous diffusion in Heisenberg models at high temperature because of nontrivial commutation relations between spin operators leading to a failure of usual hydrodynamics [9-12]. The strongest evidence for anomalous diffusion resulted from the memory function approach [9,10] and classical numerical simulations $[11,12]$. In one dimension, Heisenberg models have the additional property of being integrable [13]. As a result, at zero temperature the linear spin response is ballistic in the gapless phase [14], while at finite temperature no definite conclusion could be reached so far [15-27]. It has been argued that the regular, nonballistic contribution to spin transport can indeed be of diffusive character at finite temperature $[21,22]$.

To address this fundamental problem, we experimentally study the far-from-equilibrium dynamics of quantum spins in one and two dimensions, realized with ultracold atoms in optical lattices. In our study, we prepare initial spin spiral states of a defined wave vector and track their relaxation dynamics. Our study is also motivated by recent experiments on spin diffusion in ultracold fermions [28-30], which found an exceptionally low transverse spin diffusion constant in two dimensions [29], very different from threedimensional results [30]. These so far unexplained results motivate studies in alternative systems to check the generality of the observation. In our experiment and numerical simulations, we find that spin dynamics at high energy density in one-dimensional Heisenberg systems exhibits diffusive character. An intuitive way to understand the emergence of such a classicallike transport is given through interaction-induced dephasing between the many-body eigenstates spanning the initial spin spiral state. In contrast, the $2 \mathrm{D}$ system is shown to exhibit anomalous superdiffusion for the observed intermediate time scales, in agreement with earlier predictions [10]. We find in both cases that the closed quantum evolution at high energy density is in stark contrast to the one of a few excited magnons, which propagate ballistically [31-33].

Following the concept of spin-grating spectroscopy [34-36], we prepare initial large amplitude transverse spin spirals $|\chi(\mathbf{Q})\rangle=\prod_{j}\left(|\uparrow\rangle_{j}+e^{-i \mathbf{Q} \cdot \mathbf{x}_{j}}|\downarrow\rangle_{j}\right)$ with a controlled wave vector $\mathbf{Q}$, where $\mathbf{x}_{j}$ is the position of the lattice sites. On a phenomenological level, the evolution of the spiral would be captured through the dynamics of a single component $M_{\perp}$ of the transverse magnetization. The combination of the continuity equation and the empirical Fick's law leads to the diffusion equation $\partial M_{\perp} / \partial t=D \nabla^{2} M_{\perp}$, with a diffusion constant $D$. This equation predicts a characteristic dependence of the lifetime $\tau$ of the transverse magnetization $M_{\perp}$ on the initial wave vector $1 / \tau=D|\mathbf{Q}|^{2}$. In order to test this prediction far from equilibrium, where a vast number of states are available to scatter, we track the relaxation dynamics of the spin spiral with single-site resolution and compare our experiments to numerical simulations. 
We implement the spin Hamiltonian by using ultracold bosonic ${ }^{87} \mathrm{Rb}$ atoms in an optical lattice, initially prepared in a Mott insulating regime with unity filling. In this strong coupling regime, our atomic lattice system can be mapped to the ferromagnetic Heisenberg model [37-39], which is slightly modified in our case due to a small number of mobile particle-hole defects:

$$
\hat{H}=-J_{\mathrm{ex}} \sum_{i}\left[\frac{1}{2}\left(\hat{S}_{i}^{+} \hat{S}_{i+1}^{-}+\hat{S}_{i}^{-} \hat{S}_{i+1}^{+}\right)+\Delta \hat{S}_{i}^{z} \hat{S}_{i+1}^{z}\right]+\hat{H}_{d} .
$$

Here $J_{\text {ex }} \approx 4 J^{2} / U$ is the superexchange coupling, and $J$ and $U$ denote the hopping and interaction energy scales, respectively, of the underlying single-band Hubbard model. We note that, for the spin states employed in the experiment, the interaction energies for different spin channels vary only on the level of $1 \%$ resulting in an almost isotropic model with $\Delta \approx 1[40,41]$. The spin operators are defined through the boson creation and annihilation operators $\hat{b}_{\sigma, i}^{\dagger}$ and $\hat{b}_{\sigma, i}$ for the two spin states $\sigma=\uparrow, \downarrow$ as $\hat{S}_{i}^{+}=\hat{b}_{\uparrow, i}^{\dagger} \hat{b}_{\downarrow, i}$, $\hat{S}_{i}^{-}=\hat{b}_{\downarrow, i}^{\dagger} \hat{b}_{\uparrow, i}$, and $\hat{S}_{i}^{z}=\left(\hat{n}_{\uparrow, i}-\hat{n}_{\downarrow, i}\right) / 2$. The last term, $\hat{H}_{d}$, in Eq. (1) describes the dynamics of defects. Here we restrict the discussion to the dominating effect of holes in the Mott insulator. The probability of doubly occupied sites is assumed to be lower and thus neglected. The Hamiltonian in Eq. (1) then corresponds to the bosonic $t-J$ model [42].

Our experiments started with the preparation of a degenerate ${ }^{87} \mathrm{Rb}$ Bose gas confined in a single antinode of a vertical optical standing wave. The two-dimensional gas was then driven into the Mott insulating phase with unity filling by adiabatically switching on a horizontal square lattice with lattice spacing $a_{\text {lat }}=532 \mathrm{~nm}$. Two longlived hyperfine states $\left(|\downarrow\rangle \equiv\left|F=1, m_{F}=-1\right\rangle \quad\right.$ and $|\uparrow\rangle \equiv|2,-2\rangle)$ are used as a pseudo-spin- $1 / 2$ system. For the preparation of the initial spiral, all many-body spin dynamics was suppressed in a deep optical lattice of $20 E_{r}$ lattice depth. Here $E_{r}=h^{2} /\left(8 m a_{\text {lat }}^{2}\right)$ denotes the recoil energy of the lattice, with $m$ being the atomic mass. A global $\pi / 2$ pulse of $10 \mu$ s duration then transferred all atoms to a symmetric superposition of the two hyperfine states. Next, a relative phase between neighboring spins was imprinted by exposing the atoms to a constant magnetic field gradient of $0.2 \mathrm{G} / \mathrm{cm}$ (corresponding to a frequency shift of $20 \mathrm{~Hz} / a_{\mathrm{lat}}$ ). Time evolution in the gradient field leads to a linear growth of this relative phase over time and thus imprints a controlled spin spiral state $|\chi(\mathbf{Q})\rangle$. Subsequently, the gradient was reduced to a negligible value of $\leq 2 \mathrm{mG} / \mathrm{cm}$ for the further course of the experiment. The evolution of the strongly interacting spins was then initiated by lowering the depth of either one or both of the horizontal lattices within $5 \mathrm{~ms}$ to the desired value between 8 and $16 E_{r}$ for the experiments in $1 \mathrm{D}$ or $2 \mathrm{D}$, respectively. The experiments in $1 \mathrm{D}$ were carried out in weaker lattices, as the transition point towards the superfluid region occurs at a lower lattice depth. For the rampdown, we chose a time scale that both minimizes heating while still being short compared to the ensuing spin dynamics. Then the system was let to evolve for variable times of up to $t_{\text {hold }} \simeq 3 \hbar / J_{\text {ex }}$. For detection, the final spin configuration was frozen by rapidly increasing the lattice depth within $1 \mathrm{~ms}$ to $40 E_{r}$. A second $\pi / 2$ pulse then completed the global Ramsey interferometer by rotating the transverse spiral to the measurement basis along the $z$ direction. Finally, the $|\uparrow\rangle$ state was optically removed from the lattice, and the remaining atoms $n_{j}$ per site $j$ in the $|\downarrow\rangle$ component were imaged with single-site resolved fluorescence detection [43] (see Fig. 1).

We analyze the spiral pattern through a second-order correlation function $g_{2}(j, k)=\left\langle n_{j} n_{k}\right\rangle /\left(\left\langle n_{j}\right\rangle\left\langle n_{k}\right\rangle\right)$ and thereby avoid cancellation of the spiral signal due to shot-to-shot fluctuations in its phase caused by uniform magnetic field fluctuations. Note that in this case $g_{2}(j, k)$ is equivalent to $\operatorname{Re}\left\langle\hat{S}_{j}^{+} \hat{S}_{k}^{-}\right\rangle$when neglecting defects [41]. The correlation signal dominantly depends on the distance $d$ between sites, such that $g_{2}(d)=N \sum_{j}\left\langle n_{j} n_{j+d}\right\rangle /\left(\sum_{j}\left\langle n_{j}\right\rangle\right)^{2}$ can be used to improve the signal-to-noise ratio. Here $\langle\cdot\rangle$ represents the ensemble average over different experimental realizations, whereas the sum describes the spatial average over $N$ different positions.

For increasing times $t_{\text {hold }}$, we observe a decay of the visibility of the spiral pattern, while its period remains unchanged. An exemplary data set for such a dynamics in 1D is shown in Fig. 2 for an initial spiral with wavelength $\lambda=5.7(1) a_{\text {lat }}$. From an exponential fit to the decaying visibility, we find a lifetime of $\tau=30(3)$ ms corresponding

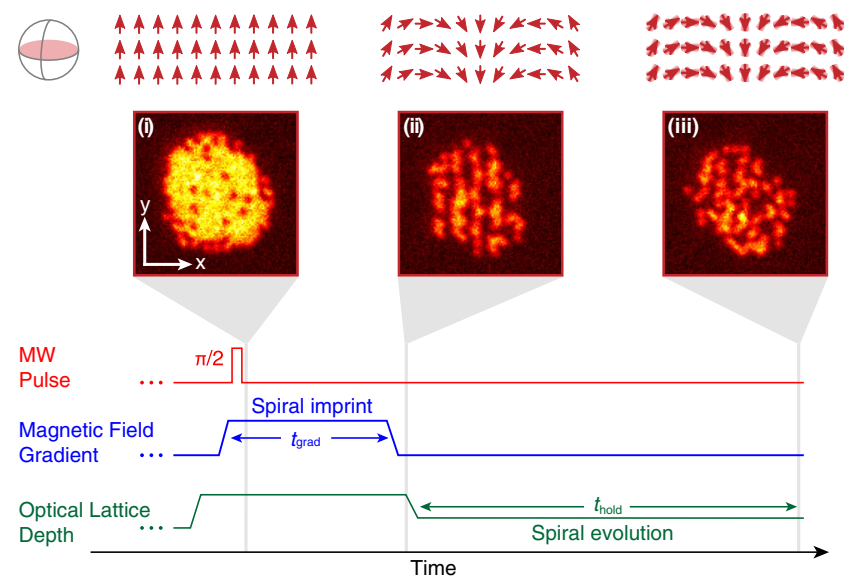

FIG. 1 (color online). Experimental sequence for the measurement of the spiral evolution. The illustrations on top show the spin distribution in the transverse plane at different stages of the experiment as indicated by the gray shading [before (i) and after (ii) spiral imprinting and after evolution (iii)]. The pictures below are single shot measurements at the respective times after an additional $\pi / 2$ pulse (not shown) and removal of the $|\uparrow\rangle$ state. The experimental sequence is depicted at the bottom. 


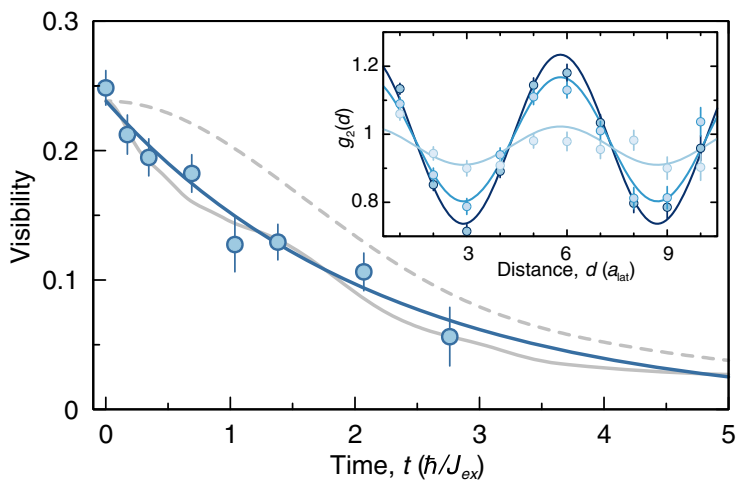

FIG. 2 (color online). Decay of a 1D spin spiral. Measured decay of an exemplary $1 \mathrm{D}$ spin spiral with wavelength $\lambda=5.7 a_{\text {lat }}$ at $10 E_{r}$ lattice depth. The solid blue line is an exponential fit used to extract the lifetime. We also show theoretical predictions of the Heisenberg model (gray, dashed line) and the $t$ - $J$ model for 0.08 hole probability (gray, solid line). The inset shows three measured correlations $g_{2}(d)$ at $t_{1}=0, t_{2}=0.7 \hbar / J_{\mathrm{ex}}$, and $t_{3}=2.8 \hbar / J_{\mathrm{ex}}$ (dark to bright blue), from which the visibility is extracted via sinusoidal fits.

to $2.1(3) \hbar / J_{\mathrm{ex}}$. We note that a simple mean-field treatment of the relaxational dynamics in the Heisenberg model based on a Landau-Lifshitz-type evolution equation does not exhibit any dynamical evolution. Thus, quantum fluctuations beyond linear order are responsible for the decay of the spin spiral. We compare the experimentally observed decay to exact diagonalization predictions for the Heisenberg and the $t-J$ model taking the nonlinearities fully into account (see Fig. 2). Both models predict an initial quadratic decay due to dephasing that happens on the fastest time scale $\left(\hbar / J_{\mathrm{ex}}\right.$ or $\left.\hbar / J\right)$ [41]. Experimentally, we only sample the decay on the superexchange time scale $\hbar / J_{\mathrm{ex}}$ and thus cannot resolve the fast initial dynamics in the $t$ - $J$ model. While both models show good qualitative agreement with the experimental data, the $t-J$ model reproduces the observations for an independently characterized hole probability of $0.08(1)$.

In order to check the assumption of diffusionlike spin transport, we measure the lifetime $\tau$ for different wave vectors $\mathbf{Q}$, in both $1 \mathrm{D}$ and $2 \mathrm{D}$. In $2 \mathrm{D}$, the spiral wave vectors were oriented diagonally to the lattice $\mathbf{Q}=(Q, Q) / \sqrt{2}$. The resulting data are shown in Fig. 3 for both dimensionalities, different lattice depths, and different initial wave vectors. When scaling the data with the exchange coupling $J_{\text {ex }}$, we find the data sets for different lattice depths to collapse. From this, we deduce that $\hbar / J_{\mathrm{ex}}$ is the relevant time scale for the main features of the observed dynamics and superexchange-mediated quantum magnetism is the underlying mechanism driving the dynamics. In order to gain further insight into the wave vector dependence of the spiral lifetime, we plot the data in a double-logarithmic plot and fit a power law with variable exponent $\alpha$ to the data $\tau \propto Q^{-\alpha}$. For our 1D data, we find an exponent of $\alpha=1.9(1)$ in good agreement with diffusive
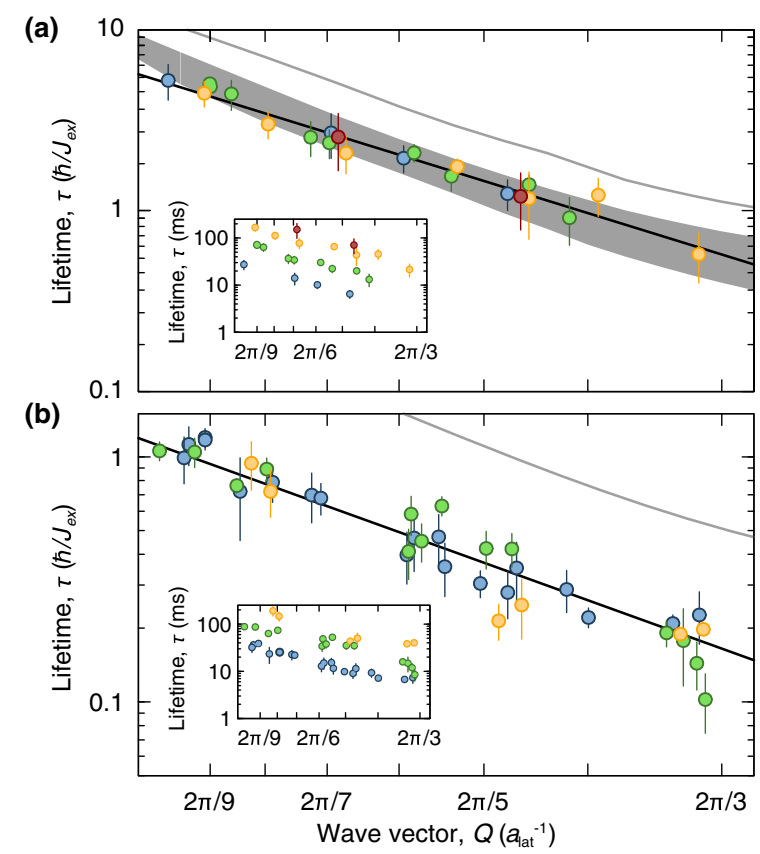

FIG. 3 (color online). Wave vector dependence of the spin spiral lifetimes. We plot the data for 1D (a) and 2D (b) spirals double logarithmically and extract the exponents via power law fits (black lines). The lifetime is scaled with the superexchange rate $\hbar / J_{\text {ex }}$, which results in a collapse of the measurements at different lattice heights [in 1D: $8 E_{r}$ (blue), $10 E_{r}$ (green), $12 E_{r}$ (yellow), and $13 E_{r}$ (red) and in 2D: $12 E_{r}$ (blue), $14 E_{r}$ (green), and $16 E_{r}$ (yellow)]. Additionally, predictions of the Heisenberg model (numerically solved in $1 \mathrm{D}$, spin wave calculations in 2D) are shown as gray solid lines. The gray band in (a) is obtained numerically from the $t$ - $J$ model with hole probabilities between 0.04 and 0.12 . The insets show the experimental data without scaling of the lifetimes.

spin transport. In 2D, the fitted exponent yields $\alpha=1.6(1)$, differing notably from the one of diffusive transport and hinting at anomalous superdiffusion. For the analysis of the data, the exchange coupling $J_{\text {ex }}$ was extracted independently from single magnon propagation measurements following our earlier results in Ref. [32]. In these measurements, we consistently find that the measured $J_{\text {ex }}$ is $20(10) \%$ larger than the one calculated from $a b$ initio single-band calculations. We attribute this difference to interaction-induced multiband effects that are expected to effectively lower $U$ but raise $J$ [44].

The observed diffusionlike behavior can be understood microscopically in the one-dimensional case, where the numerical simulations based on the Heisenberg model also point to an approximately quadratic dependence of the decay rate on the wave vector in the experimentally accessible region. As the spiral state is not an eigenstate of the Heisenberg model, it shows overlap with several many-body eigenstates. Our simulations show that the energy spread $\Delta E$ in the many-body spectrum in fact increases quadratically with the spiral wave vector $Q$ (see 




FIG. 4 (color online). Microscopic view of the diffusionlike behavior in 1D. The energy spread of eigenstates contributing to the spin spiral decay grows quadratically with wave vector $Q$. This leads to the observed quadratic $Q$ dependence of the decay rate, as expected for classical spin diffusion. The data shown for two system sizes of 12 (blue) and 16 (red) sites are obtained from full diagonalization of 1D Heisenberg chains. The insets show energy histograms weighted with the overlap of the initial spiral and the eigenstates for $Q=\pi / 4 a_{\text {lat }}$ and $Q=\pi / 2 a_{\text {lat }}$ for systems with 16 spins. A spin spiral with wave vector $Q$ is a superposition of many-body eigenstates with wave vectors $k$ that are integer multiples of $Q$.

Fig. 4). The diffusivelike behavior in the evolution of the spiral state can thus be traced back to a many-body dephasing effect, with the shortest decay time occurring for a classical Néel state $Q=\pi / a_{\text {lat }}$ (see Ref. [45]).

When comparing the prediction in detail to the experimentally measured lifetimes (see Fig. 3), we find the latter to be shifted systematically to lower values. This behavior can be reproduced when considering the $t-J$ model with the measured hole probability, indicating a good qualitative and quantitative understanding of the evolution. The observations in the 2D situation are compared to results from a spin-wave theory prediction for the case without holes [41]. While we find a similar qualitative behavior in this analysis, our experimental results are again shifted systematically towards lower lifetime values. Unfortunately, numerical simulations in 2D including holes remain currently out of reach because of the prohibitively large underlying Hilbert space.

The time scale of the diffusive behavior in 1D is set by the diffusion constant $D$. From dimensional analysis, we find its natural units to be $\hbar / \tilde{m}$, where $\tilde{m}=\hbar^{2} /\left(2 J_{\text {ex }} a_{\text {lat }}^{2}\right)$ is the effective magnon mass. When assuming diffusive behavior (fixing the exponent $\alpha=2$ ), we extract $D=$ $0.22(1) \hbar / \tilde{m}$ from our data. Remarkably, this is among the lowest values measured to date in a 1D many-body setting, even though our measurements are carried out far from equilibrium in the highly excited regime.

An intriguing additional question is the dependence of the diffusion constant on the hole density. In Fig. 5, we compare all 1D measurements for the lowest possible hole probability (the same data as shown in Fig. 3) with data

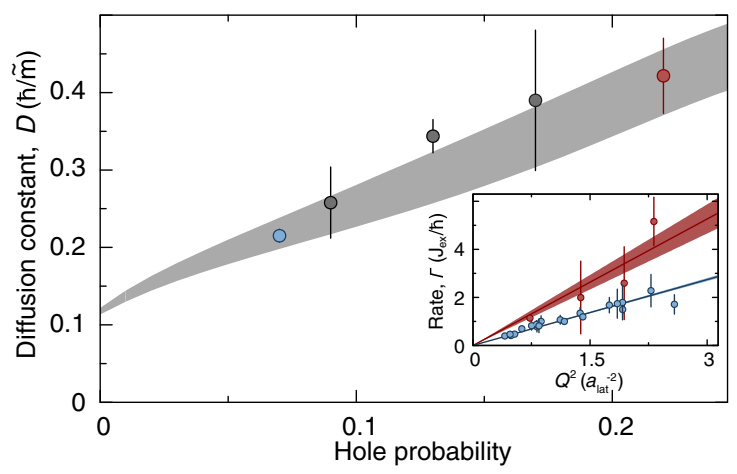

FIG. 5 (color online). Dependence of the diffusion constant in 1D on the hole density. The diffusion constant $D$ increases approximately linearly with hole probability. The gray area is the numerical result of the $t-J$ model with its $95 \%$ confidence interval. The inset shows the decay rate $1 / \tau$ of the spin spiral versus the squared wave vector $Q^{2}$ for the lowest (blue) and highest (red) hole probability.

obtained for larger hole probabilities. Our data show a clear trend towards an increasing diffusion constant with an increasing hole probability, consistent with numerical predictions based on the $t-J$ model. A linear increase can be indeed expected in 1D as each hole-localized during the preparation-introduces a fixed phase defect.

In conclusion, we have studied far-from-equilibrium spin transport in the Heisenberg model by using high-energydensity spin spiral states in 1D and 2D. A numerical analysis explained the observed diffusionlike behavior in integrable 1D chains on a microscopic level. We found that the main features of the magnetic spin transport are robust against a small number of mobile hole defects in the system. In contrast to the diffusive behavior in $1 \mathrm{D}$, we observed anomalous superdiffusion in 2D Heisenberg magnets where integrability is broken. For future studies, it would be interesting to explore the long-time behavior, which might in 1D shed light on the question of a residual ballistic transport [15-27], while in 2D it could unveil a possible crossover from a superdiffusive behavior to subdiffusive behavior [10]. Especially in 1D, it would be valuable to study spirals prepared with a wave vector close to $Q \sim \pi / a_{\text {lat }}$, where a transformation to the antiferromagnetic Heisenberg Hamiltonian is possible. Thus, one can expect that the dynamics can be described with a Luttinger liquid formalism and predictions of Refs. [21,22] could be tested. Furthermore, it would be interesting to study the absence of transport in interacting, many-body localized spin systems subject to quenched disorder [46-53] using, for instance, local interferometric techniques [54,55].

We thank I. Affleck, M. Cheneau, T. Giamarchi, F. Heidrich-Meissner, A. Läuchli, M. Lukin, and J. Thywissen for valuable discussions. The authors acknowledge support from MPG, EU (UQUAM), Harvard-MIT CUA, ARO-MURI Quism program, and ARO-MURI on Atomtronics, as well as the Austrian Science Fund (FWF) Project No. J 3361-N20. 
*Corresponding author.

shild@mpq.mpg.de

†Present address: RIKEN Center for Emergent Matter Science (CEMS), Wako, 351-0198, Japan.

[1] N. Bloembergen, Physica (Amsterdam) 15, 386 (1949).

[2] L. Van Hove, Phys. Rev. 95, 1374 (1954).

[3] P. G. De Gennes, J. Phys. Chem. Solids 4, 223 (1958).

[4] D. Hone, C. Scherer, and F. Borsa, Phys. Rev. B 9, 965 (1974).

[5] J. P. Boucher, M. A. Bakheit, M. Nechtschein, M. Villa, G. Bonera, and F. Borsa, Phys. Rev. B 13, 4098 (1976).

[6] H. Benner, Phys. Rev. B 18, 319 (1978).

[7] M. Takigawa, N. Motoyama, H. Eisaki, and S. Uchida, Phys. Rev. Lett. 76, 4612 (1996).

[8] K. R. Thurber, A. W. Hunt, T. Imai, and F. C. Chou, Phys. Rev. Lett. 87, 247202 (2001).

[9] M. Chertkov and I. Kolokolov, Phys. Rev. B 49, 3592 (1994).

[10] S. W. Lovesey, E. Engdahl, A. Cuccoli, V. Tognetti, and E. Balcar, J. Phys. Condens. Matter 6, L521 (1994).

[11] G. Müller, Phys. Rev. Lett. 60, 2785 (1988).

[12] O. F. de Alcantara Bonfim and G. Reiter, Phys. Rev. Lett. 69, 367 (1992).

[13] H. Bethe, Z. Phys. A 71, 205 (1931).

[14] B. S. Shastry and B. Sutherland, Phys. Rev. Lett. 65, 243 (1990).

[15] H. Castella, X. Zotos, and P. Prelovsek, Phys. Rev. Lett. 74, 972 (1995).

[16] X. Zotos, F. Naef, and P. Prelovsek, Phys. Rev. B 55, 11029 (1997).

[17] X. Zotos, Phys. Rev. Lett. 82, 1764 (1999).

[18] B. N. Narozhny, A. J. Millis, and N. Andrei, Phys. Rev. B 58, R2921 (1998).

[19] J. V. Alvarez and C. Gros, Phys. Rev. Lett. 88, 077203 (2002).

[20] F. Heidrich-Meisner, A. Honecker, D. C. Cabra, and W. Brenig, Phys. Rev. B 68, 134436 (2003).

[21] J. Sirker, R. G. Pereira, and I. Affleck, Phys. Rev. Lett. 103, 216602 (2009).

[22] J. Sirker, R. G. Pereira, and I. Affleck, Phys. Rev. B 83, 035115 (2011).

[23] T. Prosen, Phys. Rev. Lett. 106, 217206 (2011).

[24] M. Žnidarič, Phys. Rev. Lett. 106, 220601 (2011).

[25] R. Steinigeweg and W. Brenig, Phys. Rev. Lett. 107, 250602 (2011).

[26] C. Karrasch, J. H. Bardarson, and J. E. Moore, Phys. Rev. Lett. 108, 227206 (2012).

[27] M. Znidaric, arXiv:1405.5541.

[28] A. Sommer, M. Ku, G. Roati, and M. W. Zwierlein, Nature (London) 472, 201 (2011).

[29] M. Koschorreck, D. Pertot, E. Vogt, and M. Köhl, Nat. Phys. 9, 405 (2013).

[30] A. B. Bardon, S. Beattie, C. Luciuk, W. Cairncross, D. Fine, N. S. Cheng, G. J. A. Edge, E. Taylor, S. Zhang, S. Trotzky, and J. H. Thywissen, Science 344, 722 (2014).
[31] M. Ganahl, E. Rabel, F. H. L. Essler, and H. G. Evertz, Phys. Rev. Lett. 108, 077206 (2012).

[32] T. Fukuhara, A. Kantian, M. Endres, M. Cheneau, P. Schauß, S. Hild, D. Bellem, U. Schollwöck, T. Giamarchi, C. Gross, I. Bloch, and S. Kuhr, Nat. Phys. 9, 235 (2013).

[33] T. Fukuhara, P. Schauß, M. Endres, S. Hild, M. Cheneau, I. Bloch, and C. Gross, Nature (London) 502, 76 (2013).

[34] A. R. Cameron, P. Riblet, and A. Miller, Phys. Rev. Lett. 76, 4793 (1996).

[35] W. Zhang and D. G. Cory, Phys. Rev. Lett. 80, 1324 (1998).

[36] G. Wang, B. L. Liu, A. Balocchi, P. Renucci, C. R. Zhu, T. Amand, C. Fontaine, and X. Marie, Nat. Commun. 4, 2372 (2013).

[37] L.-M. Duan, E. Demler, and M. D. Lukin, Phys. Rev. Lett. 91, 090402 (2003).

[38] A. B. Kuklov and B. V. Svistunov, Phys. Rev. Lett. 90, 100401 (2003).

[39] E. Altman, W. Hofstetter, E. Demler, and M. D. Lukin, New J. Phys. 5, 113 (2003).

[40] D. Pertot, B. Gadway, and D. Schneble, Phys. Rev. Lett. 104, 200402 (2010).

[41] See Supplemental Material at http://link.aps.org/ supplemental/10.1103/PhysRevLett.113.147205 for additional details of experiment and theory.

[42] A. Auerbach, Interacting Electrons and Quantum Magnetism (Springer, New York, 1994).

[43] J. F. Sherson, C. Weitenberg, M. Endres, M. Cheneau, I. Bloch, and S. Kuhr, Nature (London) 467, 68 (2010).

[44] S. Will, T. Best, U. Schneider, L. Hackermüller, D.-S. Lühmann, and I. Bloch, Nature (London) 465, 197 (2010).

[45] P. Barmettler, M. Punk, V. Gritsev, E. Demler, and E. Altman, Phys. Rev. Lett. 102, 130603 (2009).

[46] D. Basko, I. Aleiner, and B. Altshuler, Ann. Phys. (Amsterdam) 321, 1126 (2006).

[47] V. Gornyi, A. D. Mirlin, and D. G. Polyakov, Phys. Rev. Lett. 95, 206603 (2005).

[48] V. Oganesyan and D. A. Huse, Phys. Rev. B 75, 155111 (2007).

[49] A. Pal and D. A. Huse, Phys. Rev. B 82, 174411 (2010).

[50] J. H. Bardarson, F. Pollmann, and J. E. Moore, Phys. Rev. Lett. 109, 017202 (2012).

[51] R. Vosk and E. Altman, Phys. Rev. Lett. 110, 067204 (2013).

[52] V. Oganesyan and D. A. Huse, arXiv:1305.4915.

[53] M. Serbyn, Z. Papić, and D. A. Abanin, Phys. Rev. Lett. 111, 127201 (2013).

[54] M. Knap, A. Kantian, T. Giamarchi, I. Bloch, M. D. Lukin, and E. Demler, Phys. Rev. Lett. 111, 147205 (2013).

[55] M. Serbyn, M. Knap, S. Gopalakrishnan, Z. Papić, N. Y. Yao, C. R. Laumann, D. A. Abanin, M. D. Lukin, and E. A. Demler, Phys. Rev. Lett. 113, 147204 (2014). 